\title{
Calibration of echosounder using standard target method
}

\author{
Liuqing Yang ${ }^{*}$, Yi Chen, and Jun Zhang \\ Hangzhou Applied Acoustics Research Institute, 311400, P.R.China
}

\begin{abstract}
In this work, we develop an underwater echosounder and use the standard target method to calibrate the performance of the device. In the calibration experiments, a solid tungsten carbide sphere of $38 \mathrm{~mm}$ diameter is used as a standard target for calibrating a HPCTB-200-35 echosounder (manufactured by Hangzhou Applied Acoustics Research Institute) with a working frequency of 220 kHz. Further, the measurement data and uncertainty are presented and analyzed; these results demonstrate that the standard target method can calibrate the combined transmitting-receiving response of echosounders effectively. In our calibration experiment, the combined transmitting-receiving response of HPCTB-200-35 is about $33.8 \mathrm{~dB}$, and the measurement uncertainty is about $1.0 \mathrm{~dB}(k=2)$.
\end{abstract}

\section{Introduction}

Echosounder is a type of active sonar which uses transducers to emit sound waves in the water and receive signals reflected back from obstacles. The distance between transducer and obstacle can be calculated according to the propagation time and the speed of sound. From the working principle of the echosounder, the accuracy of the measurement results is directly affected by the sound source level and receiving sensitivity. To ensure the reliability and stability of the measurement results, the echosounder must be calibrated. It is also believed by the International Organization for Standardization (ISO) that "Without calibration, the sonar remains a piece of equipment; with calibration, it is elevated to the status of an instrument." [1]

At present, the acoustic parameters of active sonar mainly include sound source level, transmitting voltage response, transmitting directivity, receiving sensitivity, and receiving directivity etc. There are two main methods used for calibrating these parameters, the standard hydrophone method and the standard target method. Standard hydrophone method is widely used in calibration of sonar performance, which uses a standard hydrophone and its measurement system to measure the active sonar performance of transmitting and receiving, respectively. Unlike standard hydrophone method, the standard target method uses a target with known acoustic properties to measure the transmitting-receiving response of the active sonar based on the active sonar equation. Transmitting-receiving response is always used to assess the performance of active sonar, like maximum range etc.

In 1967, Robert B. Patterson [2] simultaneously measured transmitting-receiving response of the transducer using the water surface as a total reflector. *Corresponding author: yanglqathz@163.com
This method could be used in calibration of active sonar, but the condition of total reflection was too strict and ideal. Therefore designing and selecting a standard target was a key part of active sonar calibration. In 1972, V. G. Welsby and J. E. Hudson [3] proposed that ping pong ball, whose target strength can be calculated through acoustic model, could be used as a target for calibrating active sonars. In 1981, Louis R. Dragonette [4] verified the acoustic scattering model of solid elastic sphere proposed by Robert Hicking [5] in 1964. The experimental results were basically consistent with the theoretical calculation results. Latterly, elastic spheres were widely used for calibrating active sonars or transducers. Since 1982, Kenneth G. Foote [6-8] has designed a series of standard targets for active sonar calibration.

The standard target method was established for calibrating active sonar by Kenneth G. Foote [9] in 1987. Then it was widely used and developed, for instance, Dezhang Chu, etc [10] used the method to calibrate the multibeam sonar SM2000/90kHz which worked on the frequency of $90 \mathrm{kHz}$. A. Islas-Cital[11] also calibrated the phase of wide-band sonar in the same way. In 2015, the calibration methods of different active sonars through standard target [11] were discussed and published at the International Council for the Exploration of the Sea (ICES). However, these researchers usually only consider the computational uncertainty of the standard target or do not analyze the type A and B uncertainty of the measurement.

This paper studies the calibration of echosounder HPCTB-200-35 which was manufactured by Hangzhou Applied Acoustics Research Institute (HARRI) and working on the frequency of $220 \mathrm{kHz}$. 
A standard target of a solid tungsten carbide sphere with diameter of $\$ 38 \mathrm{~mm}$ is used for calibration, and the measurement uncertainty is analyzed in detail.

\section{Calibration principle of active sonar}

The coordinate system for the active sonar transducer is shown in Fig.1, and the reference center of transducer sound center is placed on the origin point. The distance between reference center of transducer and target is $r$, target is a solid sphere with radius a and it locates at the direction $(\theta, \varphi)$ in the far-field of the transducer and target. The incident pressure which emits from the transducer can be expressed as

$$
p_{i}=\frac{p_{t}}{r} e^{i(\omega t-k r)}
$$

where $p_{\mathrm{i}}$ is the incident wave's sound pressure, $p_{\mathrm{t}}$ is the amplitude, $\omega$ is the acoustic angular frequency, $k$ is wave -number, $t$ is the time, $i=\sqrt{-1}$.

If the harmonic time and phase are not considered, the equation (1) can be expressed as,

$$
p_{i}=\frac{p_{t}}{r}
$$

and the scattered sound pressure is expressed as

$$
p_{r}=\frac{p_{i} f_{b s}}{r}=\frac{p_{t} f_{b s}}{r^{2}}
$$

where $f_{\mathrm{bs}}$ is the amplitude of backscattering function, and $T S=20 \lg f_{\mathrm{bs}}, T S$ is target strength.

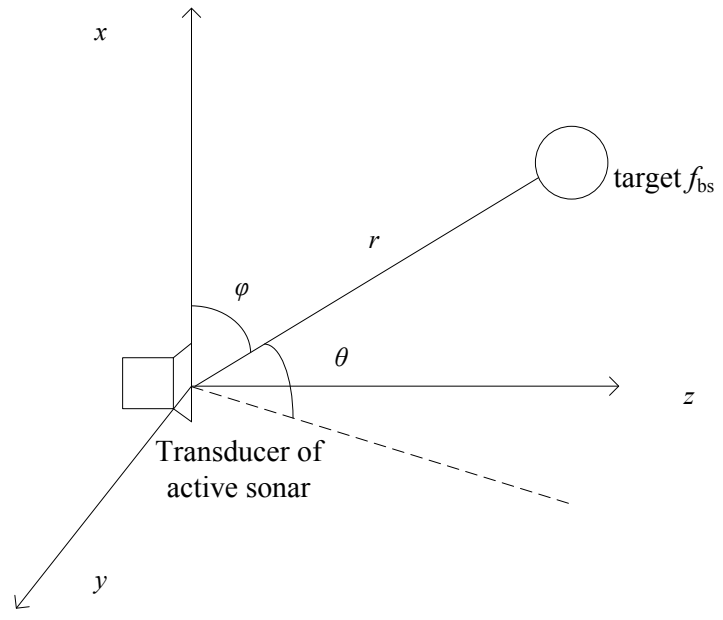

Fig. 1. Diagram of target and active sonar transducer in coordinate system.

There is a relationship between the receiving sound pressure and transmitting voltage (or current) of transducer

$$
\left\{\begin{array}{l}
p_{t}=U_{t} S_{v} d_{1}(\theta, \varphi) \\
p_{r}=\frac{U_{r}}{M d_{2}(\theta, \varphi)}
\end{array}\right.
$$

where $U_{\mathrm{t}}$ is transmitting voltage, $d_{1}(\theta, \varphi)$ is transmitting directivity function, $S_{\mathrm{v}}$ is transmitting voltage response, $U_{\mathrm{r}}$ is receiving voltage, $d_{2}(\theta, \varphi)$ is receiving directivity function, $M$ is receiving voltage response.

Now, the equation (3) can be expressed as

$$
\begin{aligned}
U_{r} & =\frac{U_{t} S_{v} M}{r^{2}} f_{b s} d_{1}(\theta, \varphi) d_{2}(\theta, \varphi) \\
& =\frac{U_{t} S_{v} M}{r^{2}} f_{b s} b(\theta, \varphi)
\end{aligned}
$$

where $b(\theta, \varphi)$ is the product of two-way directivity. For self-reciprocity transducer, $d_{1}(\theta, \varphi)=d_{2}(\theta, \varphi)$, $b(\theta, \varphi)=d^{2}{ }_{1}(\theta, \varphi)$ is the directivity pattern. When target locates in the on-axis of transducer, namely $\theta=0, \varphi=0, b(0,0)=1$, then the equation (5) could be expressed as

$$
\boldsymbol{S}_{v}+\boldsymbol{M}=20 \lg \left(U_{r}\right)-20 \lg \left(U_{t}\right)-T S+40 \lg (r)
$$

where $S_{V}$ is transmitting voltage response expressed in $\mathrm{dB}($ re $1 \mu \mathrm{Pa} \cdot \mathrm{m} / \mathrm{V}), \boldsymbol{M}$ is receiving sensitivity expressed in $\mathrm{dB}($ re $1 \mathrm{~V} / \mu \mathrm{Pa})$. From equation (6), transmitting-receiving response of active sonar could be obtained when $U_{\mathrm{t}}$ and $U_{\mathrm{r}}$ are measured. Because the transmitting voltage of echosounder is a constant, then calibration process could be described in Fig. 2 . echosounder is regarded as black box, signal processing is not considered, the equation (6) is expressed as

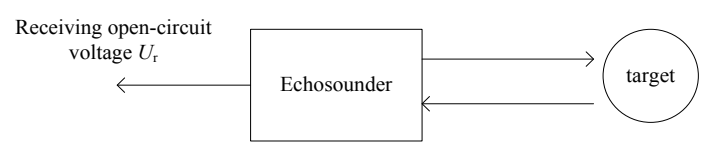

Fig. 2. Calibration process of echosounder.

$$
S L+\boldsymbol{M}=20 \lg \left(U_{r}\right)-T S+40 \lg (r)
$$

where $S L$ is source level in $\mathrm{dB} . S L+\boldsymbol{M}$ represents combined transmitting- receiving response of echosounder. We use $C R$ to represent this variable, namely

$$
C R=20 \lg \left(U_{r}\right)-T S+40 \lg (r)
$$

\section{Echosounder and the design of the standard target}

\subsection{Echosounder to be calibrated}

The echosounder to be calibrated here is HPCTB-200 -35 which is manufactured by HAARI. It is used to measure the depth of water or distance between two objects in shallow water, and its maximum range is about $100 \mathrm{~m}$. HPCTB-200-35 consists of transducer, $10 \mathrm{~m}$ length cable and circuit block, where circuit block included emitting signal, digital signal to analog signal, power amplifier, receiving signal, receiving gain and analog signal to digital signal. Transducer was a circular piston projector with diameter of $35 \mathrm{~mm}$, and operation its frequency was $220 \mathrm{kHz}$. 


\subsection{Design of the standard target}

To be a standard for calibrating HPCTB-200-35 echosounder, the target should satisfy following requirements, such as the material of target needs to be pure, and the target needs to be freely located, as well as the target strength should lie within certain limits.

In order to accurately estimate the acoustic scattering of target, the material of target must be pure. Tungsten carbide (6\% cobalt binder) consists of $94 \%$ $\mathrm{WC}$ and $6 \%$ cobalt binder. In addition, tungsten carbide with $6 \%$ cobalt binder has been proved insensitivity of acoustic scattering properties to environmental variations by Kenneth G. Foote [7].This property is beneficial to the calibration of HPCTB-200-35.

Due to the spherically symmetrical, scattering of spherical target is independent of target orientation. Therefore a solid tungsten carbide sphere is used to be a standard target for calibrating.

According to the requirements of HPCTB-200-35 echosounder and experiences of the experiment, the nominal target strength of $-40 \mathrm{~dB}[6-8]$ is sought, which value is large enough to lie well clear of background noise. Therefore, a solid sphere of tungsten carbide with diameter $\Phi 38 \mathrm{~mm}$ is selected for calibration of HPCTB-200-35. Its target strength is $-40.03 \mathrm{~dB}(@ 220 \mathrm{kHz})$, and its size is a common size for industry.

\section{Calibration system of echosounder}

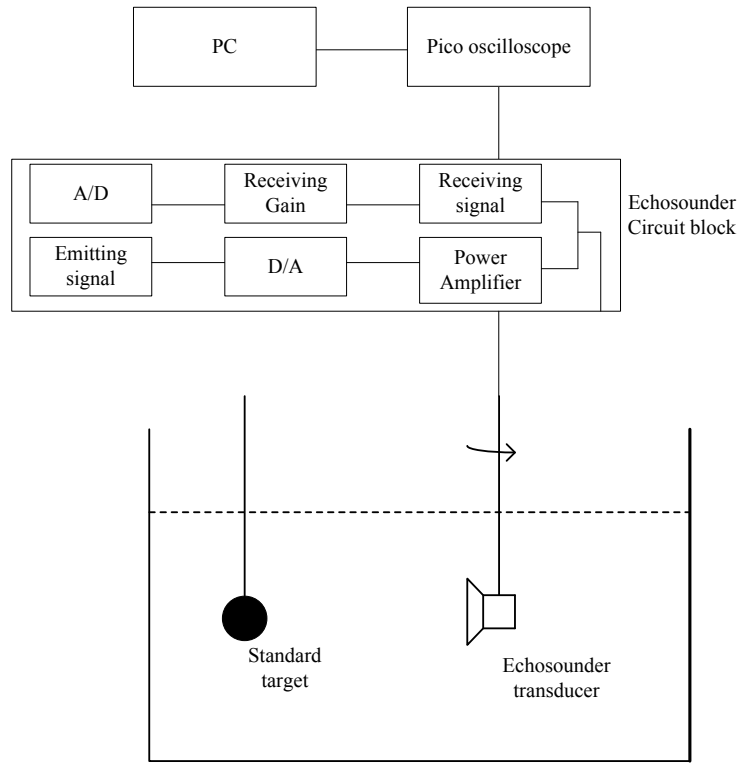

Fig. 3. Calibration diagram of HPCTB-200-35 echosounder. Calibration experiment was performed in a water tank with dimension $1.8 \mathrm{~m} \times 1.8 \mathrm{~m} \times 2 \mathrm{~m}$. The calibration diagram of HPCTB-200-35 echosounder was shown as Fig.3. It consists of PC computer, Pico oscilloscope, echosounder, solid tungsten carbide sphere with diameter $38 \mathrm{~mm}$ as standard target.

The transducer should be mounted at a moving platform which could be rotated as well as vertically and horizontally moved. The standard target was suspended at a fixed distance from the mounted transducer, and was located on sound axis of transducer for HPCTB-200-35 echosounder. The standard target and transducer were on the far-field of each other, and the echo voltages were recorded at least three times for average. Here, we use equation (8) to calculate the combined transmitting-receiving response.

\section{Calibration results and analysis of measurement uncertainty}

\subsection{Calibration results and discussion}

When calibration of $C R$, the transmitting signal duration of echosounder is $0.3 \mathrm{~ms}$, center frequency is $220 \mathrm{kHz}$. Eight times experiments were performed at eight different distances, and the results are listed in table 1 .

Table 1. Comparison of measurement results of $C R$ in different distances.

\begin{tabular}{|c|c|c|}
\hline $\boldsymbol{r} / \mathbf{c m}$ & $\boldsymbol{U}_{\mathbf{r}} / \mathbf{V p p}$ & $\boldsymbol{C R} / \mathbf{d B}$ \\
\hline 50 & 5.495 & 33.72 \\
\hline 55 & 4.795 & 34.20 \\
\hline 60 & 3.872 & 33.86 \\
\hline 65 & 3.341 & 33.97 \\
\hline 70 & 2.797 & 33.72 \\
\hline 75 & 2.465 & 33.82 \\
\hline 80 & 2.172 & 33.85 \\
\hline 85 & 1.901 & 33.75 \\
\hline
\end{tabular}

It can be seen from the Table 1, the calibration of combined transmitting-receiving response $C R$ of HPCTB-200-35 is $33.8 \mathrm{~dB}$, and the results of measurement are not irrelative with the measurement distance between transducer and target. In order to verify the accuracy of the calibration result, the standard hydrophone method was used in HARRA to get the sensitivity and transmitting voltage response of transducer of echosounder. The sensitivity and transmitting voltage response were $-176.7 \mathrm{~dB}$ (re $1 \mathrm{~V} / \mu \mathrm{Pa} @ 220 \mathrm{kHz}), 161.4 \mathrm{~dB}(\mathrm{re} 1 \mu \mathrm{Pa} \cdot \mathrm{m} / \mathrm{V} @ 220 \mathrm{kHz})$ respectively. At that time the mean square root value of transmitting voltage was about $277.3 \mathrm{~V}$. Therefore, $C R$ of calibrated using standard hydrophone method was $33.6 \mathrm{~dB}$; the deviation between these two methods is only $0.2 \mathrm{~dB}$. The value is substantial agreement with standard target method.

\subsection{Analysis of measurement uncertainty}

Measurement result has a close relation with the calibration result. Considering all the variables of equation (8) which affect the measurement results, the combined uncertainty can be expressed as 
$u_{c}(C R)=\sqrt{\left(\frac{\partial C R}{\partial U_{r}}\right)^{2} u_{c}^{2}\left(U_{r}\right)+\left(\frac{\partial C R}{\partial T S}\right)^{2} u_{c}^{2}(T S)+\left(\frac{\partial C R}{\partial r}\right)^{2} u_{c}^{2}(r)}$

Because target strength TS of solid sphere is determined by itself and environment, including material density $\rho$, longitudinal sound speed $c_{1}$ and transverse sound speed $c_{2}$ of material, deviations of diameter $d$, density $\rho_{1}$ and temperature $T$ of medium etc. These variables should be considered in calculation of the uncertainty of standard target. The calculation acoustic model of target strength here we use is from Robert Hickling [13], and the relation information is given in the Table 2.

The uncertainty of $T S$ can be expressed as

$$
u_{c}(T S)=\max _{1 \leq i \leq 6}\left(u_{i}(T S)\right)=0.2 \mathrm{~dB}
$$

The combined uncertainties of $U_{\mathrm{r}}$ and $r$ are calculated by the equation (10), which can be expressed as

$$
u_{c}=\sqrt{u_{A}^{2}+u_{B}^{2}}
$$

where $u_{\mathrm{A}}$ is the measurement uncertainty of type $\mathrm{A} ; u_{\mathrm{B}}$ is the measurement uncertainty of type B.

In the calibration experiment of HPCTB-200-35, $u_{\mathrm{A}}\left(U_{\mathrm{r}}\right)$ is $0.0671 \mathrm{~V}, u_{\mathrm{B}}\left(U_{\mathrm{r}}\right)$ is $0.080 \mathrm{~V}, u_{\mathrm{A}}(r)$ is $0.00 \mathrm{~m}$ and $u_{\mathrm{B}}(r)$ is $0.005 \mathrm{~m}$. And $u_{\mathrm{c}}\left(U_{\mathrm{r}}\right)$ and $u_{\mathrm{c}}(r)$ can be calculated by equation (10), the values are $0.0955 \mathrm{~V}$, and $0.006 \mathrm{~m}$ respectively.

\begin{tabular}{|c|c|c|c|c|c|c|}
\hline Variables & $\begin{array}{c}\rho \\
\left(14900 \pm 31 \mathrm{~kg} / \mathrm{m}^{3}\right)\end{array}$ & $\begin{array}{c}c_{1} \\
(6853 \pm 80 \mathrm{~m} / \mathrm{s})\end{array}$ & $\begin{array}{c}c_{2} \\
(4171 \pm 50 \mathrm{~m} / \mathrm{s})\end{array}$ & $T\left(18 \pm 1^{\circ} \mathrm{C}\right)$ & $\begin{array}{c}d \\
(38 \pm 0.1 \mathrm{~mm})\end{array}$ & $\begin{array}{c}\rho_{1} \\
(1000 \pm 20 \\
\left.\mathrm{kg} / \mathrm{m}^{3}\right) \\
\end{array}$ \\
\hline $\begin{array}{c}\text { Uncertainty } \\
u(\mathrm{~dB})\end{array}$ & 0.04 & 0.2 & 0.12 & 0.1 & 0.05 & 0.01 \\
\hline
\end{tabular}

Table 2. Parameter and its uncertainties used in calculation of TS.

On the basis of these work, he combined transmitting-receiving response $C R$ can be calculated from equation (9), calculating combined uncertainty of the combined transmitting-receiving response. The value is

$$
u_{c}(C R) \approx 0.5 \mathrm{~dB}
$$

nd the expended uncertainty $u$ of the confidence probability of $95 \%(k=2)$ is

$$
u=2 u_{c}(C R) \approx 1.0 \mathrm{~dB}
$$

\section{Conclusions}

The standard target method can be efficiently used for calibrating combined transmitting-receiving $C R$ of echosounder. This had been proved in calibration experiment of HPCTB-200-35 echosounder working on the frequency of $220 \mathrm{kHz}$. The $C R$ of HPCTB-200-35 calibrated is about $33.8 \mathrm{~dB}$, and expended uncertainty is $1.0 \mathrm{~dB}$. The calibration results of using standard target method have been verified by standard hydrophone method in HAARI. Accurate calibration results are beneficial to the design, assessments and improvement of HPCTB200-35 echosounder.

This work was founded by Chinese National Key Research and Development Program 2016 YFF0200900.

\section{References}

1. ISO TC43 SC3 WG4. Underwater acoustics standard-target method of calibrating active sonars for imaging and measuring scattering (working draft). (2017).

2. R. B. Patterson. J. Acoust. Soc. Am., 13(4): 653-655 (1967).

3. V. G. Welsby and J. E. Hudson, Journal of Sound and Vibration, 20(3): 399-406 (1972).
4. L. R. Dragonette, S. K. Numrich, L. J. Frank, J. Acoust. Soc. Am. 69(4): 1186-1189 (1981).

5. Robert Hicking, J. Acoust. Soc. Am., 36(6): 1124-1137 (1964).

6. K. G. Foote, J. Acoust. Soc. Am., 71(3): 742-747 (1982).

7. K. G. Foote, J. Acoust. Soc. Am., 73(3): 1054-1063( 1983).

8. K. G. Foote, J. Acoust. Soc. Am., 75(2):612-616 (1984).

9. K. G. Foote, H. P. Knudsen, G. Vestnes, et al. ICES Coop.Res. Rep, no.144,69 (1987).

10. Dezhang Chu. L. A. Mayer, IEEE, 1633-1636., (2003).

11. A. Islas-Cital, P. R. Atkins, K.Y. Foo, IEEE, (2010).

12. D. A. Demer et.al. ICES Coop. Res. Rep., no.326, 133 (2015).

13. Robert Hickling, J. Acoust. Soc. Am., 34(10): 1582-1592 (1962). 